\title{
Generation of Tetrafluoroethylene-Propylene Elastomer-Based Microfluidic Devices for Drug Toxicity and Metabolism Studies
}

\author{
Emi Sano, Sayaka Deguchi, Naoki Matsuoka, Masahiro Tsuda, Mengyang Wang, Kaori Kosugi, \\ Chihiro Mori, Keisuke Yagi, Aya Wada, Shinsuke Yamasaki, Tsuyoshi Kawai, Masahide Yodogawa, \\ Hiroyuki Mizuguchi, Norihito Nakazawa, Fumiyoshi Yamashita, Yu-suke Torisawa,* \\ and Kazuo Takayama*
}

Cite This: ACS Omega 2021, 6, 24859-24865

Read Online

ACCESS | W Metrics \& More 回 Article Recommendations

Supporting Information

ABSTRACT: Polydimethylsiloxane (PDMS) is widely used to fabricate microfluidic organs-on-chips. Using these devices (PDMS-based devices), the mechanical microenvironment of living tissues, such as pulmonary respiration and intestinal peristalsis, can be reproduced in vitro. However, the use of PDMS-based devices in drug discovery research is limited because of their extensive absorption of drugs. In this study, we investigated the feasibility of the tetrafluoroethylene-propylene (FEPM) elastomer to fabricate a hepatocyte-on-a-chip (FEPM-based hepatocyte chip) with lower drug absorption. The FEPM-based hepatocyte chip expressed drug-metabolizing enzymes, drugconjugating enzymes, and drug transporters. Also, it could produce

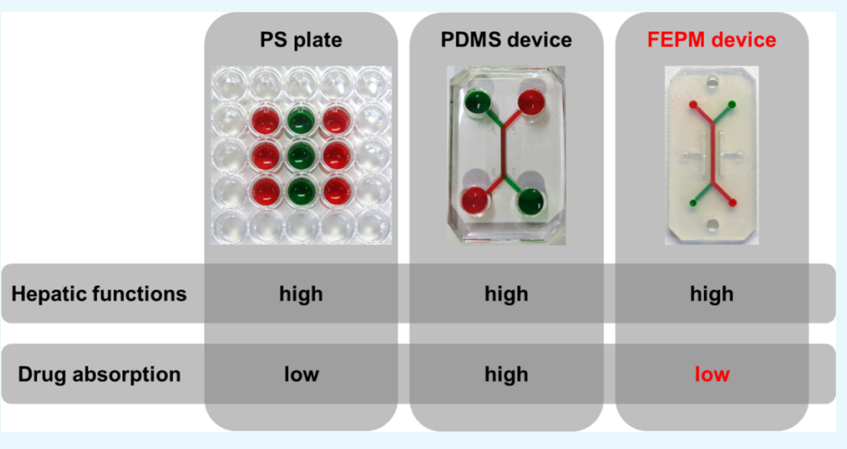
human albumin. Although the metabolites of midazolam and bufuralol were hardly detected in the PDMS-based hepatocyte chip, they were detected abundantly in the FEPM-based hepatocyte chip. Finally, coumarin-induced hepatocyte cytotoxicity was less severe in the PDMS-based hepatocyte chip than in the FEPM-based hepatocyte chip, reflecting the different drug absorptions of the two chips. In conclusion, the FEPM-based hepatocyte chip could be a useful tool in drug discovery research, including drug metabolism and toxicity studies.

\section{INTRODUCTION}

Organ-on-a-chip technology is promising for the early stage of drug discovery research. ${ }^{1}$ By culturing cells in a microfluidic device, this technology can reproduce the dynamic threedimensional environments of cells in the living body. Fluid shear stress and mechanical strain partially support the reconstitution of the organ functions. ${ }^{2}$ Cell-cell interactions can also be reproduced by seeding different cells in multiple channels. ${ }^{3}$ Thus, an organ-on-a-chip brings a different light to drug discovery research by evaluating drug candidate compounds under much more physiological conditions than conventional two-dimensional culture systems.

The microfluidic device used in an organ-on-a-chip is often made of polydimethylsiloxane (PDMS) because PDMS is a colorless, inexpensive, and easily moldable material. ${ }^{3-6}$ Furthermore, because the elastic nature of PDMS gives high actuation stretching ability to the microfluidic device, organ movements, such as pulmonary respiration and intestinal peristalsis, can be reproduced. ${ }^{2,7}$ However, PDMS has the defect of absorbing small hydrophobic molecules. ${ }^{8}$ To reduce the absorption of small hydrophobic molecules into the microfluidic devices, some non-PDMS materials were used, but there are still limitations. Although the absorption of a fluorescent dye, Nile red, can be decreased using glass-based microfluidic devices, ${ }^{9}$ they were difficult to stretch. In addition, the chemical absorption can be avoided using UV ozonetreated polycarbonate or cyclic olefin copolymer-based microfluidic devices, ${ }^{10}$ but they were also difficult to stretch. Development of microfluidic devices based on a rubber material that can avoid chemical absorption is desired.

In this context, we have succeeded in developing microfluidic devices using the tetrafluoroethylene-propylene (FEPM) elastomer, a rubber material that hardly absorbs small hydrophobic molecules, and collagen vitrigel (VIT), a vitrified thin collagen gel membrane that facilitates threedimensional cell culture. ${ }^{11,12}$ FEPM has excellent heat resistance, chemical resistance, and oil resistance. In addition, the VIT membrane has excellent material permeability, high

Received: July 14, 2021

Published: September 15, 2021 

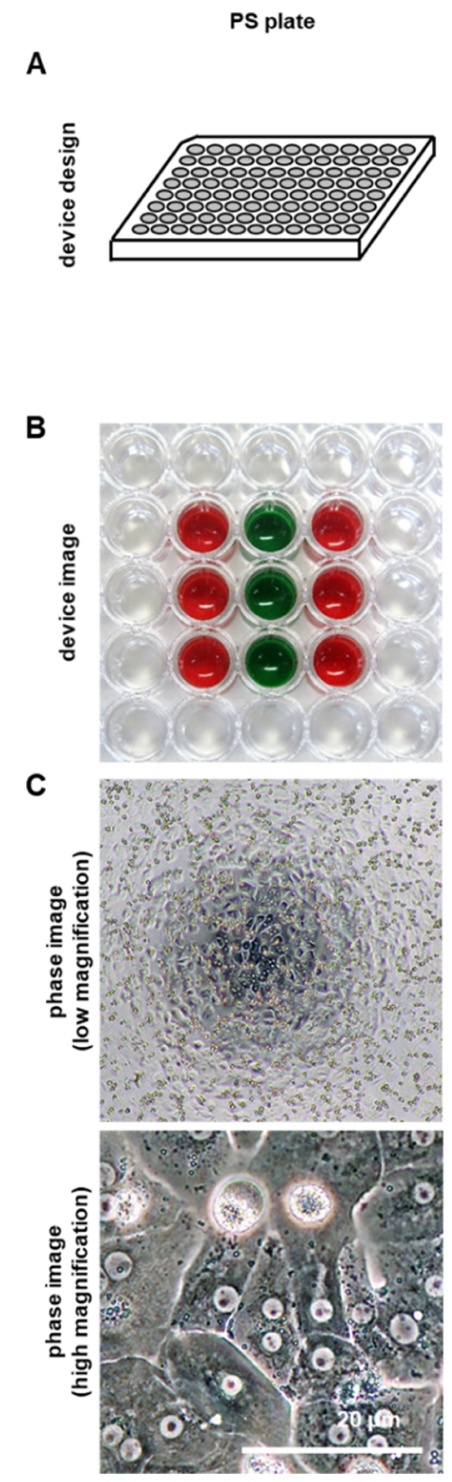
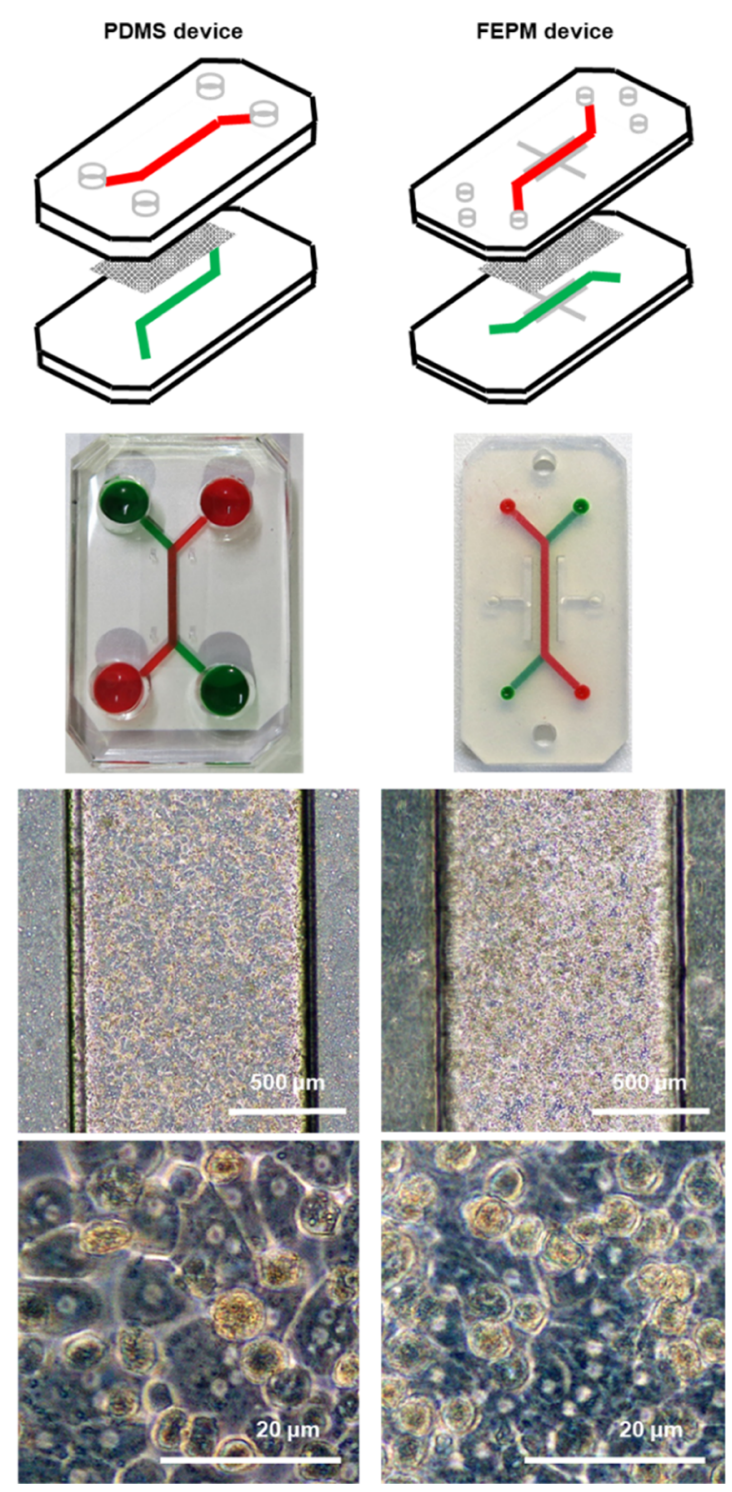

Figure 1. PHH cultured in the PDMS-based and FEPM-based devices. (A,B) Design (A) and photos (B) of the PS plate and PDMS-based and FEPM-based devices. (C) PHHs were seeded in the PS plate or injected into the PDMS-based or FEPM-based device. Phase images of the PHH cultured in each device.

transparency, and strength ${ }^{12,13}$ and is normally used without extracellular matrix coating, which is required for PDMS-based microfluidic devices (PDMS-based devices). We previously demonstrated that small hydrophobic molecules including rhodamine B, nifedipine, Bay K8644, and coumarin are less likely to be absorbed by FEPM-based microfluidic devices (FEPM-based devices) than PDMS-based microfluidic devices. However, we have not examined whether the cellular response induced by small hydrophobic molecules can be evaluated using a FEPM-based organ-on-a-chip. In this study, we developed a FEPM-based hepatocyte-on-a-chip (hepatocyte chip) with human hepatocytes and investigated whether drug discovery assays including drug metabolism and drug toxicity could be performed.

\section{RESULTS}

Primary Human Hepatocytes Cultured in PDMS- and FEPM-Based Devices. Our FEPM-based and PDMS-based devices consist of two microchannels separated by a VIT membrane and a PET membrane, respectively. The top and bottom channels are shown as red and green, respectively, in Figure 1A and B (Figure 1). Primary human hepatocytes (PHHs) were seeded into the top channel of the two devices (Figure 1C, top). Polygonal and binuclear PHHs could be observed in both devices (Figure 1C, bottom). However, compared with the polystyrene (PS) multiwell plate (PS plate), it was difficult to observe the cell morphology in the two devices.

Gene Expression and Protein Expression Analyses of Hepatic Markers. We investigated whether the hepatic functions of $\mathrm{PHH}$ are affected by the device material. The gene expression levels of hepatocyte markers were examined in PHH cultured in the PS plate, PDMS-based device, and FEPM-based device. There were no significant differences in the gene expression levels of typical hepatic markers (Figure 2A), drug-metabolizing enzymes [cytochrome P450 (CYP) families, Figure $2 \mathrm{~B}$ ] or drug-conjugating enzymes, and drug transporters (Figure 2C) between the three systems. The amount of human albumin (ALB) secreted in the medium was also not different (Figure 3A), and immunostaining analysis 

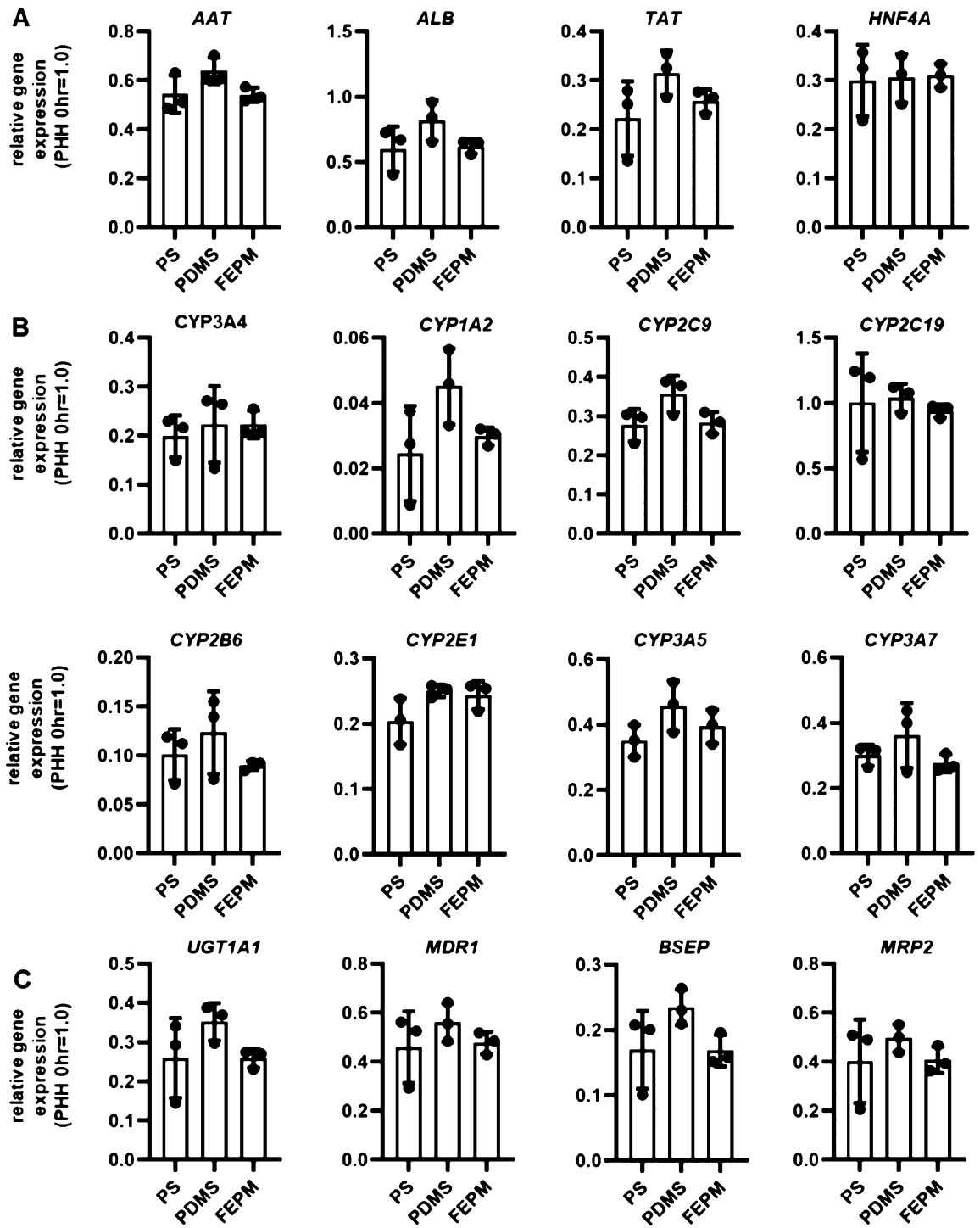

Figure 2. Gene expression analysis of hepatic markers. PHHs were cultured in the PS plate, PDMS-based device, and FEPM-based device for $24 \mathrm{~h}$. The gene expression levels of general hepatic markers (A), CYPs (B), and drug-conjugating enzymes and transporters (C) were examined by qPCR analysis. The expressions are normalized to the gene expression levels of PHHs immediately after thawing (PHH $0 \mathrm{~h}$ ). All data are represented as means $\pm \mathrm{SD}(n=3)$.

showed that PHHs strongly expressed hepatic markers [ALB, cytokeratin 18 (CK18), CYP3A4, alpha-1 antitrypsin (AAT), and hepatocyte nuclear factor 4 alpha (HNF4A)] (Figure 3B). These results suggest that PHHs cultured in the FEPM-based device phenotypically resemble PHHs cultured in the PS plate and PDMS-based device.

Drug Toxicity and Metabolism Tests Using PDMSand FEPM-Based Hepatocyte Chips. We have previously reported that the FEPM-based device is less likely to absorb small hydrophobic molecules than the PDMS-based device. ${ }^{11}$ Here, we examined whether the FEPM-based hepatocyte chip could be used for drug toxicity and drug metabolism tests.

First, a hepatotoxicity test using coumarin, which is easily absorbed by PDMS-based devices, was performed in the FEPM-based hepatocyte chip. Coumarin is contained in many plants, and its derivatives are used as drugs. However, the excessive intake of coumarin induces liver injury. ${ }^{14}$ We thus exposed PHHs cultured on the PS plate, PDMS-based device, and FEPM-based device to various concentrations of coumarin and measured the cell viability (Figure 4A). We found that the cell viability of PHHs in the FEPM-based device and on the PS plate was about the same but significantly higher in the PDMSbased device. This result suggests that coumarin-induced hepatocyte toxicity is less detectable in PHHs cultured in the PDMS-based device.

Next, a drug metabolism test was performed using midazolam (MDZ) and bufuralol (BUF). MDZ and BUF are widely used drugs for measuring CYP3A4 and CYP2D6 activities, respectively. After injecting medium containing MDZ and BUF into the PDMS-based and FEPM-based hepatocyte chips, the amounts of MDZ, BUF, and their metabolites in the medium were measured. Over time, the concentration of MDZ in the PDMS-based device decreased to $5.23 \%$ that on the PS plate, but the concentration in the FEPM-based device was maintained to at least $86.2 \%$ (Figure 4B). Regarding BUF, the concentrations were 2.18 and $37.2 \%$, respectively (Figure 4B). These results suggest that the FEPMbased device is less likely to absorb MDZ or BUF than the 
A

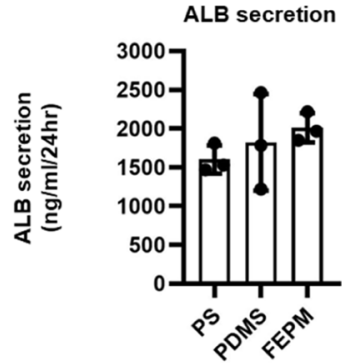

B
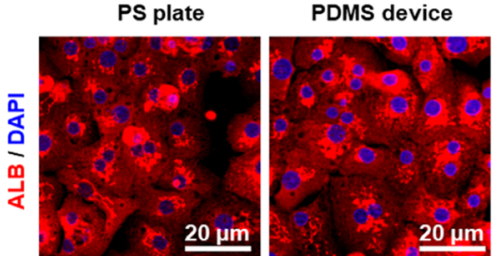

FEPM device
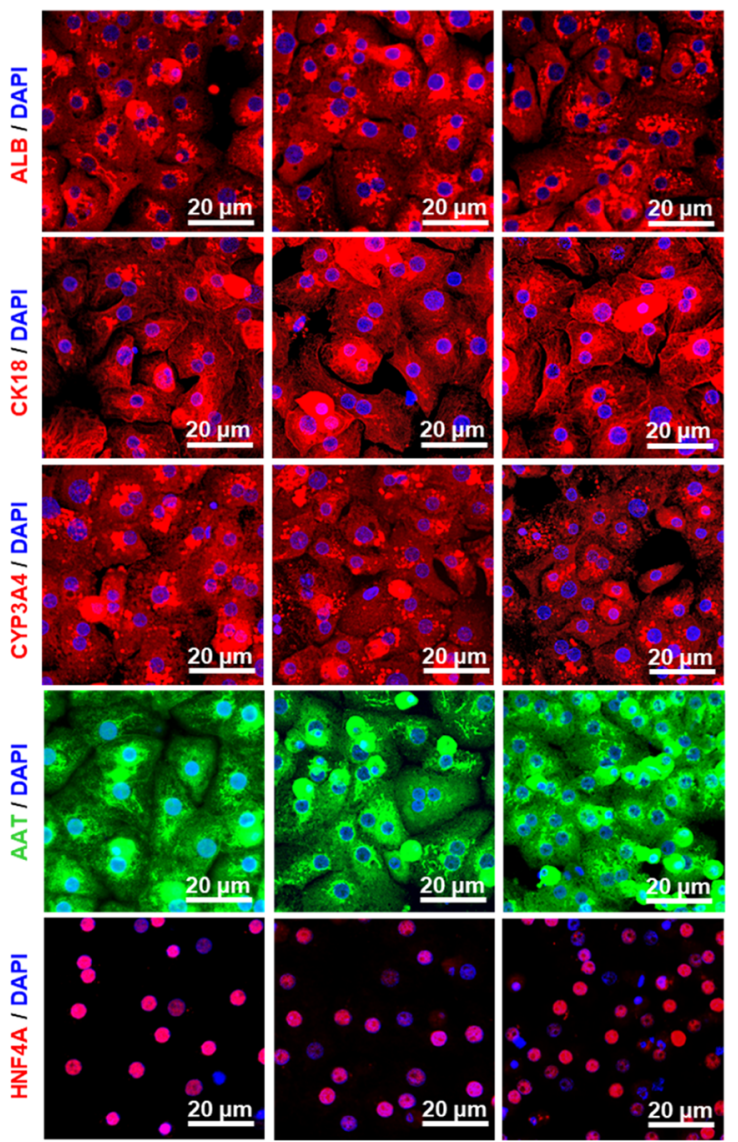

Figure 3. Protein expression analysis of hepatic markers. PHHs were cultured in the PS plate, PDMS-based device, and FEPM-based device for $24 \mathrm{~h}$. (A) Average amounts of ALB secretion in top and bottom channels were examined by ELISA. All data are represented as means $\pm \mathrm{SD}(n=3)$. (B) Immunocytochemical analysis of ALB (red), CK18 (red), CYP3A4 (red), AAT (green), and HNF4A (red) in cultured PHHs. Nuclei were counterstained with 4',6-diamidino-2-phenylindole (DAPI) (blue).

PDMS-based device. As for the metabolizes, the cumulative amount of 1 '-hydroxymidazolam (1OH-MDZ), the CYP3A4 metabolite of MDZ, produced by PHHs in the PDMS-based device decreased to only $2.16 \%$ that on the PS plate but to $30.38 \%$ in the FEPM-based device (Figure 4C). Similarly, the amount of $1^{\prime}$-hydroxybufuralol (1OH-BUF) was 14.75 and $69.9 \%$, respectively (Figure 4C). These results suggest that the metabolites of small hydrophobic molecules are significantly easier to detect with the FEPM-based hepatocyte chip than the PDMS-based hepatocyte chip.
A

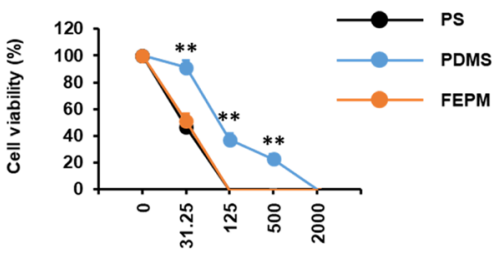

Concentration of coumarin $(\mu \mathrm{M})$

B
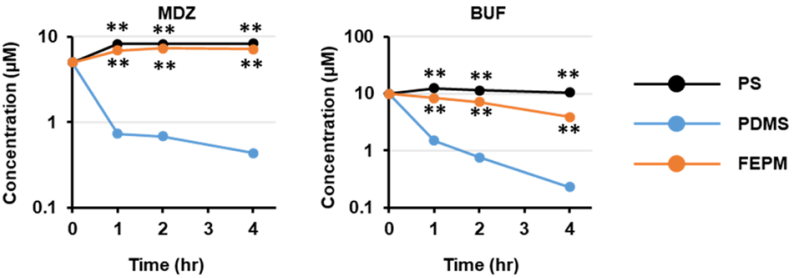

C

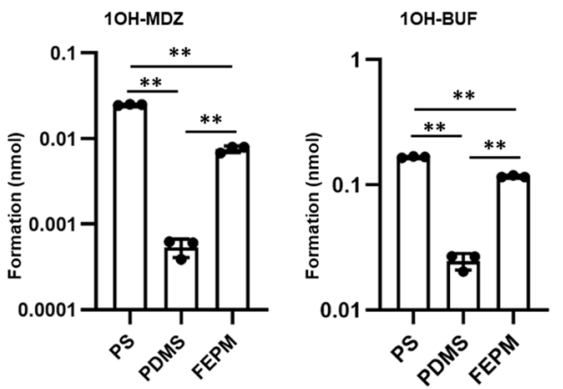

Figure 4. Drug toxicity and metabolism tests using the PDMS-based and FEPM-based hepatocyte chips. PHHs were cultured in the PS plate, PDMS-based device, and FEPM-based device for $24 \mathrm{~h}$. (A) $\mathrm{PHHs}$ were exposed to various concentrations of coumarin for 4 days. Then, the cell viability was examined by the WST- 8 assay and normalized to the percentage of cells treated with the vehicle only. Statistical significance was evaluated by two-way analysis of variance (ANOVA) followed by Tukey's post-hoc test $(* * p<0.01)$. (B) CYPmediated drug-metabolizing capacities of $\mathrm{PHHs}$ were evaluated by quantifying MDZ (the substrate of CYP3A4), BUF (the substrate of CYP2D6), and their metabolites. Medium containing MDZ and BUF was injected into the PS plate, PDMS-based device, and FEPM-based device. The average quantity of these drugs in top and bottom channels was measured by LC-MS/MS. Statistical significance was evaluated by two-way ANOVA followed by Bonferroni's post-hoc test $(* * p<0.01$, compared with the PDMS-based device). (C) Additionally, the average quantity of their metabolites (1OH-MDZ and $1 \mathrm{OH}-\mathrm{BUF}$, respectively) in top and bottom channels was measured by LC-MS/MS. Statistical significance was evaluated by one-way ANOVA followed by Tukey's post-hoc test $(* * p<0.01)$. All data are represented as means $\pm \mathrm{SD}(n=3)$.

\section{DISCUSSION}

In this study, we generated the FEPM-based hepatocyte chip, which consists of PHHs and a FEPM-based device, and then examined whether it could be used for drug discovery research including drug metabolism and drug toxicity. There was no difference in the hepatocyte function of PHHs cultured on the PS plate and in the PDMS-based device or FEPM-based device. In contrast, the results of the drug toxicity and drug metabolism tests were different between the FEPM-based and PDMS-based hepatocyte chips. The FEPM-based device could detect coumarin-induced hepatotoxicity at higher sensitivity, presumably due to the lower drug absorption compared with the PDMS-based device. ${ }^{11}$ Additionally, the amount of MDZ and BUF metabolites was much higher in the FEPM-based 
hepatocyte chip because the concentration of the parental drugs was maintained during the experiment (1OH-MDZ: $0.00054 \mathrm{nmol}$ in the PDMS-based device and $0.00755 \mathrm{nmol}$ in the FEPM-based device; 1OH-BUF: $0.025 \mathrm{nmol}$ in the PDMSbased device and $0.117 \mathrm{nmol}$ in the FEPM-based device). These results suggest that hepatocytes cultured in the two devices have similar functions but different drug absorptions.

Coumarin is a natural compound contained in cinnamon, but its excessive intake can cause liver injury. ${ }^{14}$ Coumarininduced hepatotoxicity is known to be more severe in rats than in humans. This is because in humans, about $70 \%$ of coumarin is metabolized into harmless 7-hydroxycoumarin, while in rats, most of the coumarin is metabolized into toxic 3,4-coumarin epoxide. $^{15,16}$ Considering the report by Jang et al. that the species-specific toxicities of bosentan could be recapitulated using liver chips, ${ }^{3}$ in the future, we plan to investigate species differences in coumarin-induced hepatotoxicity using the FEPM-based hepatocyte chip.

Microfluidic devices using other elastomer materials that poorly absorb small hydrophobic molecules have also been developed. ${ }^{17}$ Domansky et al. fabricated a microfluidic device using styrene-ethylenebutylene-styrene (SEBS-based device) ${ }^{18}$ However, the absorption rate of coumarin in PDMSbased and SEBS-based devices was 71 and $76 \%$, respectively, suggesting that both devices absorb coumarin to the same degree. On the other hand, they also tested the absorption rate of rhodamine B, finding that it was $74 \%$ with the PDMS-based device but $53 \%$ with the SEBS-based device. We have previously reported that our FEPM-based device hardly absorbs rhodamine B and coumarin. ${ }^{11}$ Although the present study confirmed the low absorbance of the FEPM-based device with two other drugs (MDZ and BUF), the number of tested drugs is still limited. Further studies are necessary before widely applying FEPM-based device to drug discovery research.

\section{CONCLUSIONS}

In this study, we generated a FEPM-based hepatocyte chip and confirmed its applicability to drug metabolism and drug toxicity tests. Because the small intestine also plays an important role in drug absorption and metabolism, our findings suggest that the development of a FEPM-based intestine chip would benefit drug discovery research. In the near future, we plan to develop an in vitro model that can evaluate the first-pass effect using FEPM-based liver and intestine chips. Because of the low drug absorption, we expect that the organ-on-a-chip technology fabricated with FEPM will contribute to drug discovery research.

\section{MATERIALS AND METHODS}

PDMS-Based Devices. The microfluidic device consists of two layers of microchannels separated by a semipermeable membrane. The microchannel layers were fabricated from PDMS using a soft lithographic method. A PDMS prepolymer (Sylgard 184, Dow Corning) at a ratio of 10:1 base to curing agent was cast against a mold composed of SU-8 2150 (MicroChem) patterns formed on a silicon wafer. The crosssectional size of the microchannels was $1 \mathrm{~mm}$ in width and 300 $\mu \mathrm{m}$ in height. To introduce solutions into the microchannels, access holes were punched through the PDMS using a $6 \mathrm{~mm}$ biopsy punch (Kai Corporation). Two PDMS layers were bonded to a semipermeable PET membrane containing $3.0 \mu \mathrm{m}$ pores (\#353091 Falcon) using a thin layer of liquid PDMS prepolymer as mortar. ${ }^{19}$ The PDMS prepolymer was spincoated (4000 rpm for $60 \mathrm{~s}$ ) onto a glass slide. Subsequently, both the top and bottom channel layers were placed on the glass slide to transfer the thin layer of the PDMS prepolymer onto the embossed PDMS surfaces. The membrane was then placed onto the bottom layer and sandwiched with the top layer. The combined layers were left at room temperature for 1 day to remove air bubbles and then put into an oven at $60{ }^{\circ} \mathrm{C}$ overnight to cure the PDMS glue. The PDMS devices were sterilized by placing them under UV light for $1 \mathrm{~h}$ prior to the cell culture.

FEPM-Based Devices and Adapters. The microfluidic devices consist of two layers of microchannels separated by a thin VIT membrane. In addition to the devices, we fabricated a culture medium reservoir as adapters to culture cells without any perfusion system. The microchannel layers and a $2 \mathrm{~mm}$ thick sheet for the adapters were fabricated from FEPM compounds (AFLAS, AGC Inc.) at a ratio of 10:4 base to vulcanizing agents at $60{ }^{\circ} \mathrm{C}$ on a two-roll mill $(\phi 811 \times 1811$ test roll machine for chemical machine design and production, Yamatetsu Machinery, Inc.). Each layer was formed by compression molding with a custom-designed hard chromeplated two-layer mold on a 200-ton vacuum compression molding machine (TYC-V-2RT, Tung Yu Hydraulic Machinery Co., Ltd.), press-curing at $160{ }^{\circ} \mathrm{C}$ for $30 \mathrm{~min}$, and postcuring at $200{ }^{\circ} \mathrm{C}$ for $2 \mathrm{~h}$ in an oven (DH612, Yamato Scientific Co., Ltd.). The cross-sectional size of the microchannels is 1 $\mathrm{mm}$ width $\times 1 \mathrm{~mm}$ height, and the gaps between each microchannel are $1 \mathrm{~mm}$. A $10 \mu \mathrm{m}$-thick VIT membrane (VITC001, AGC Techno Glass Co., Ltd.) was placed between the two channel layers. The FEPM layers and the VIT membrane were bonded together for $5 \mathrm{~s}$ at room temperature using a selfadhesion system that was generated during the formation of each FEPM layer, allowing the channel layers to be assembled without the use of glue. The bonding strength of the FEPM device containing the membrane was measured using a digital spring scale (PS-01, Dr. meter) and a vertical drill guide (DS70, SK11) by pulling the FEPM layer vertically until it detached $(n=5)$. The dimensions of the FEPM layer were 20 $\mathrm{mm}$ (width) $\times 40 \mathrm{~mm}$ (length) $\times 2 \mathrm{~mm}$ (thickness). Each adapter was made with a $2 \mathrm{~mm}$-thick sheet using a punching die and a processing machine [OP-23(H)-55, Akebono Machines Industries Co., Ltd.]. The adapters were $10 \mathrm{~mm} \times$ $20 \mathrm{~mm}$ in size with two $6 \mathrm{~mm} \varphi$ holes. The FEPM microfluidic devices and adapters were sterilized by placing them under UV light for $2 \mathrm{~h}$ prior to the cell culture.

PHHs Cultured on PS Plates or PDMS and FEPM Devices. Cryopreserved human hepatocytes (Lonza) were used in this study. The vials of PHHs were rapidly thawed in a shaking water bath at $37^{\circ} \mathrm{C}$, the contents of each vial were emptied in pre-warmed cryopreserved hepatocyte recovery medium (Thermo Fisher Scientific), and the suspension was centrifuged at $1200 \mathrm{rpm}$ for $5 \mathrm{~min}$ at room temperature. The $\mathrm{PHH}$ was suspended at $5 \times 10^{6}$ cells $/ \mathrm{mL}$ in HCM (Lonza) containing $10 \%$ fetal calf serum (FCS). For the PS plates, the $\mathrm{PHH}$ was seeded at $1.0 \times 10^{5}$ cells $/ \mathrm{cm}^{2}$ in HCM (Lonza) containing $10 \%$ FCS onto type I collagen-coated 96-well plates. For the PDMS devices, the devices were coated with type I collagen before $10 \mu \mathrm{L}$ of cell suspension was injected. For the FEPM devices, $20 \mu \mathrm{L}$ of cell suspension was injected without type I collagen coating. After $1 \mathrm{~h}, 200 \mu \mathrm{L}$ of medium was added into each of the top and bottom channels of the 
devices. PHHs, which were cultured $24 \mathrm{~h}$ after the plating, were used in the experiments.

Quantitative PCR. ISOGEN (NIPPON GENE) was used to isolate total RNA from the cells. A Superscript VILO cDNA synthesis kit (Thermo Fisher Scientific) was used to synthesize cDNA from the isolated total RNA. Quantitative RT-PCR was performed with SYBR Green PCR Master Mix (Thermo Fisher Scientific) using a StepOnePlus qPCR system (Thermo Fisher Scientific). The $2^{-\Delta \Delta \mathrm{CT}}$ method was adopted for the relative quantitation of target mRNA levels. The values of the target genes were normalized by those of the housekeeping gene, glyceraldehyde 3-phosphate dehydrogenase (GAPDH). The PCR primer sequences are summarized in Supporting Information Table S1.

ALB Secretion. To evaluate the human ALB secretion capacity of the $\mathrm{PHH}$, the culture supernatants, which were incubated for $24 \mathrm{~h}$ after the culture medium was added, were collected and analyzed using the human albumin ELISA quantitation set (Bethyl Laboratories). ELISA was performed according to the manufacturer's instructions.

Immunohistochemistry. PHHs were fixed with $4 \%$ paraformaldehyde in PBS for $15 \mathrm{~min}$. After blocking the cells with PBS containing $10 \%$ FCS, $1 \%$ bovine serum albumin, and $0.2 \%$ Triton $\mathrm{X}-100$ at room temperature for $45 \mathrm{~min}$, the cells were incubated with a primary antibody at $4{ }^{\circ} \mathrm{C}$ overnight and then with a secondary antibody at room temperature for $1 \mathrm{~h}$. All antibodies used in this report are described in Supporting Information Table S2.

Assessment of Coumarin-Induced Cell Toxicity. PHHs were exposed to coumarin (FUJIFILM Wako) for 4 days. The cell viability was examined by the WST- 8 assay, which was performed using a cell counting kit- 8 purchased from DOJINDO LABORATORIES according to the manufacturer's instructions. The cell viability was calculated as the percentage of cells with no treatment.

Assay for CYP Activity. To examine the CYP activity, LC-MS/MS analysis was performed. Thirty minutes before the CYP substrate treatment, the culture medium of $\mathrm{PHH}$ was replaced with Dulbecco's modified Eagle's medium (DMEM) containing $10 \mathrm{mM}$ HEPES. Then, PHHs were cultured with medium containing $5 \mu \mathrm{M} \mathrm{MDZ} \mathrm{(a} \mathrm{substrate} \mathrm{whose} \mathrm{metabolite}$ is $1 \mathrm{OH}-\mathrm{MDZ}$ ) and $10 \mu \mathrm{M}$ BUF (a substrate whose metabolite is $1 \mathrm{OH}-\mathrm{BUF}$ ). A $100 \mu \mathrm{L}$ aliquot was collected at 1,2 , and $4 \mathrm{~h}$ after treatment with the substrates, and the loss was compensated with an equal volume of fresh substratecontaining medium. The collected samples were mixed with the same volume of acetonitrile (FUJIFILM Wako) containing $5 \mu \mathrm{M}$ propranolol (an internal standard). The mixtures were filtered with a Cosmonice Filter W (Nacalai Tesque) with a pore size of $0.45 \mu \mathrm{m}$, and then, the supernatant was analyzed by LC-MS/MS using a LCMS-8040 (Shimadzu). Detailed information about the ionization mode and multiple-reaction monitoring (MRM) transition of the mass spectrometer is summarized in Supporting Information Table S3. The dwell time for each MRM transition was set at $100 \mathrm{msec}$. LC separations were carried out at $40{ }^{\circ} \mathrm{C}$ with an $\mathrm{XBridge} \mathrm{BEH}$ C18 column, $1.7 \mu \mathrm{m}, 2.1 \times 50 \mathrm{~mm}$ (Waters). The mobilephase conditions are summarized in Supporting Information Table S4. The concentrations of the substrates and their metabolites were measured according to the standard curve.

Statistical Analyses. Statistical significance was evaluated by one-way or two-way analysis of variance (ANOVA) followed by Tukey's or Bonferroni's post-hoc test. Details are described in the figure legends.

\section{ASSOCIATED CONTENT}

\section{Supporting Information}

The Supporting Information is available free of charge at https://pubs.acs.org/doi/10.1021/acsomega.1c03719.

List of the primers and antibodies and the information of LC-MS/MS analysis (PDF)

\section{AUTHOR INFORMATION}

\section{Corresponding Authors}

Yu-suke Torisawa - Department of Micro Engineering, Kyoto University, Kyoto 615-8540, Japan;

Email: yusuketorisawa@gmail.com

Kazuo Takayama - Center for iPS Cell Research and Application (CiRA), Kyoto University, Kyoto 606-8507, Japan; 이이이.org/0000-0002-1132-2457; Email: kazuo.takayama@cira.kyoto-u.ac.jp

\section{Authors}

Emi Sano - Center for iPS Cell Research and Application (CiRA), Kyoto University, Kyoto 606-8507, Japan; Department of Micro Engineering, Kyoto University, Kyoto 615-8540, Japan

Sayaka Deguchi - Center for iPS Cell Research and Application (CiRA), Kyoto University, Kyoto 606-8507, Japan; Laboratory of Biochemistry and Molecular Biology, Graduate School of Pharmaceutical Sciences, Osaka University, Osaka 565-0871, Japan

Naoki Matsuoka - AGC Inc., Tokyo 100-8405, Japan

Masahiro Tsuda - Department of Applied Pharmaceutics and Pharmacokinetics, Graduate School of Pharmaceutical Sciences, Kyoto University, Kyoto 606-8501, Japan

Mengyang Wang - Department of Applied Pharmaceutics and Pharmacokinetics, Graduate School of Pharmaceutical Sciences, Kyoto University, Kyoto 606-8501, Japan

Kaori Kosugi - Center for iPS Cell Research and Application (CiRA), Kyoto University, Kyoto 606-8507, Japan; Department of Micro Engineering, Kyoto University, Kyoto 615-8540, Japan

Chihiro Mori - Department of Micro Engineering, Kyoto University, Kyoto 615-8540, Japan

Keisuke Yagi - AGC Inc., Tokyo 100-8405, Japan

Aya Wada - AGC Inc., Tokyo 100-8405, Japan

Shinsuke Yamasaki - AGC Inc., Tokyo 100-8405, Japan

Tsuyoshi Kawai - AGC Inc., Tokyo 100-8405, Japan

Masahide Yodogawa - AGC Inc., Tokyo 100-8405, Japan

Hiroyuki Mizuguchi - Laboratory of Biochemistry and Molecular Biology, Graduate School of Pharmaceutical Sciences, Osaka University, Osaka 565-0871, Japan

Norihito Nakazawa - AGC Inc., Tokyo 100-8405, Japan

Fumiyoshi Yamashita - Department of Applied Pharmaceutics and Pharmacokinetics, Graduate School of Pharmaceutical Sciences and Department of Drug Delivery Research, Graduate School of Pharmaceutical Sciences, Kyoto University, Kyoto 606-8501, Japan

Complete contact information is available at: https://pubs.acs.org/10.1021/acsomega.1c03719 


\section{Author Contributions}

E.S.: human hepatocyte culture and analysis of hepatic functions. S.D.: manuscript writing, drug-metabolizing analysis, and statistical analysis. N.M.: FEPM device fabrication and quality inspection and manuscript writing. M.T.: drugmetabolizing analysis. W.M.: drug-metabolizing analysis. K.K.: human hepatocyte culture and generating the PDMS device. C.M.: human hepatocyte culture. K.Y.: FEPM device fabrication and quality inspection. A.W.: FEPM device fabrication and quality inspection. S.Y.: FEPM device fabrication and quality inspection. T.K.: FEPM device fabrication and quality inspection. M.Y.: FEPM device fabrication and quality inspection. H.M.: discussion. N.N.: FEPM device fabrication and quality inspection. Fumiyoshi Yamashita: drug-metabolizing analysis. Y.-s.T.: research design, generating the PDMS device, and manuscript writing. K.T.: research design, manuscript writing, and final approval

\section{Notes}

The authors declare the following competing financial interest(s): N.M., K.Y., A.W., S.Y., T.K., M.Y., and N.N. are employees of AGC Inc. and AGC Group Companies.

${ }^{\mathrm{I}}$ E.S. and S.D. are co-first authors.

The data that support the findings of this study are available from the corresponding authors upon reasonable request.

\section{ACKNOWLEDGMENTS}

We thank Natsumi Mimura and Ayaka Sakamoto (Kyoto University) for technical assistance with the human hepatocyte culture and hepatic function analysis. We also thank Dr. Peter Karagiannis (Kyoto University) for critical reading of the manuscript. We thank Naoki Shizuka, Kazuo Iino, Michio Jingu, Yu-ki Kitamura, Shigeo Suetomi, Tomonori Sato, Tomomi Hagiwara, Motoya Murase, Kana Tanabe, Toshiyuki Meguro, Takeshi Yamada, Osamu Karigome, Dr. Salvekar Abhijit, Toshio Miki, and Momoe Kubota, (AGC, Inc., and its associate companies) for the FEPM device fabrication and quality inspection. This research was supported by grants from the Japan Agency for Medical Research and Development (AMED) (21be0304202h0005) and the iPS Cell Research Fund.

\section{REFERENCES}

(1) Ewart, L.; Dehne, E.-M.; Fabre, K.; Gibbs, S.; Hickman, J.; Hornberg, E.; Ingelman-Sundberg, M.; Jang, K.-J.; Jones, D. R.; Lauschke, V. M.; Marx, U.; Mettetal, J. T.; Pointon, A.; Williams, D.; Zimmermann, W.-H.; Newham, P. Application of microphysiological systems to enhance safety assessment in drug discovery. Annu. Rev. Pharmacol. Toxicol. 2018, 58, 65-82.

(2) Grassart, A.; Malardé, V.; Gobaa, S.; Sartori-Rupp, A.; Kerns, J.; Karalis, K.; Marteyn, B.; Sansonetti, P.; Sauvonnet, N. Bioengineered human organ-on-chip reveals intestinal microenvironment and mechanical forces impacting Shigella infection. Cell Host Microbe 2019, 26, 435-444.

(3) Jang, K.-J.; Otieno, M. A.; Ronxhi, J.; Lim, H.-K.; Ewart, L.; Kodella, K. R.; Petropolis, D. B.; Kulkarni, G.; Rubins, J. E.; Conegliano, D.; Nawroth, J.; Simic, D.; Lam, W.; Singer, M.; Barale, E.; Singh, B.; Sonee, M.; Streeter, A. J.; Manthey, C.; Jones, B.; Srivastava, A.; Andersson, L. C.; Williams, D.; Park, H.; Barrile, R.; Sliz, J.; Herland, A.; Haney, S.; Karalis, K.; Ingber, D. E.; Hamilton, G. A. Reproducing human and cross-species drug toxicities using a LiverChip. Sci. Transl. Med. 2019, 11, aax5516.

(4) Huh, D.; Hamilton, G. A.; Ingber, D. E. From 3D cell culture to organs-on-chips. Trends Cell Biol. 2011, 21, 745-754.
(5) Foster, A. J.; Chouhan, B.; Regan, S. L.; Rollison, H.; Amberntsson, S.; Andersson, L. C.; Srivastava, A.; Darnell, M.; Cairns, J.; Lazic, S. E.; Jang, K.-J.; Petropolis, D. B.; Kodella, K.; Rubins, J. E.; Williams, D.; Hamilton, G. A.; Ewart, L.; Morgan, P. Integrated in vitro models for hepatic safety and metabolism: evaluation of a human Liver-Chip and liver spheroid. Arch. Toxicol. 2019, 93, 1021-1037.

(6) Deguchi, S.; Tsuda, M.; Kosugi, K.; Sakamoto, A.; Mimura, N.; Negoro, R.; Sano, E.; Nobe, T.; Maeda, K.; Kusuhara, H.; Mizuguchi, H.; Yamashita, F.; Torisawa, Y.-s.; Takayama, K. Usability of Polydimethylsiloxane-Based Microfluidic Devices in Pharmaceutical Research Using Human Hepatocytes. ACS Biomater. Sci. Eng. 2021, 7, 3648-3657.

(7) Huh, D.; Matthews, B. D.; Mammoto, A.; Montoya-Zavala, M.; Hsin, H. Y.; Ingber, D. E. Reconstituting organ-level lung functions on a chip. Science 2010, 328, 1662-1668.

(8) Wang, J. D.; Douville, N. J.; Takayama, S.; ElSayed, M. Quantitative analysis of molecular absorption into PDMS microfluidic channels. Ann. Biomed. Eng. 2012, 40, 1862-1873.

(9) Hirama, H.; Satoh, T.; Sugiura, S.; Shin, K.; Onuki-Nagasaki, R.; Kanamori, T.; Inoue, T. Glass-based organ-on-a-chip device for restricting small molecular absorption. J. Biosci. Bioeng. 2019, 127, 641-646.

(10) van Midwoud, P. M.; Janse, A.; Merema, M. T.; Groothuis, G. M. M.; Verpoorte, E. Comparison of biocompatibility and adsorption properties of different plastics for advanced microfluidic cell and tissue culture models. Anal. Chem. 2012, 84, 3938-3944.

(11) Sano, E.; Mori, C.; Matsuoka, N.; Ozaki, Y.; Yagi, K.; Wada, A.; Tashima, K.; Yamasaki, S.; Tanabe, K.; Yano, K.; Torisawa, Y.-s. Tetrafluoroethylene-propylene elastomer for fabrication of microfluidic organs-on-chips resistant to drug absorption. Micromachines 2019, 10, 793.

(12) Takezawa, T.; Ozaki, K.; Nitani, A.; Takabayashi, C.; ShimoOka, T. Collagen vitrigel: a novel scaffold that can facilitate a threedimensional culture for reconstructing organoids. Cell Transplant. 2004, 13, 463-474.

(13) Takezawa, T.; Nitani, A.; Shimo-Oka, T.; Takayama, Y. A protein-permeable scaffold of a collagen vitrigel membrane useful for reconstructing crosstalk models between two different cell types. Cells Tissues Organs 2007, 185, 237-241.

(14) Abraham, K.; Wöhrlin, F.; Lindtner, O.; Heinemeyer, G.; Lampen, A. Toxicology and risk assessment of coumarin: focus on human data. Mol. Nutr. Food Res. 2010, 54, 228-239.

(15) Pearce, R.; Greenway, D.; Parkinson, A. Species differences and interindividual variation in liver microsomal cytochrome $\mathrm{P} 4502 \mathrm{~A}$ enzymes: effects on coumarin, dicumarol, and testosterone oxidation. Arch. Biochem. Biophys. 1992, 298, 211-225.

(16) Vassallo, J. D.; Hicks, S. M.; Daston, G. P.; LehmanMcKeeman, L. D. Metabolic detoxification determines species differences in coumarin-induced hepatotoxicity. Toxicol. Sci. 2004, $80,249-257$.

(17) Campbell, S. B.; Wu, Q.; Yazbeck, J.; Liu, C.; Okhovatian, S.; Radisic, M. Beyond Polydimethylsiloxane: Alternative Materials for Fabrication of Organ-on-a-Chip Devices and Microphysiological Systems. ACS Biomater. Sci. Eng. 2021, 7, 2880-2899.

(18) Domansky, K.; Sliz, J. D.; Wen, N.; Hinojosa, C.; Thompson, G.; Fraser, J. P.; Hamkins-Indik, T.; Hamilton, G. A.; Levner, D.; Ingber, D. E. SEBS elastomers for fabrication of microfluidic devices with reduced drug absorption by injection molding and extrusion. Microfluid. Nanofluid. 2017, 21, 107.

(19) Chueh, B.-h.; Huh, D.; Kyrtsos, C. R.; Houssin, T.; Futai, N.; Takayama, S. Leakage-free bonding of porous membranes into layered microfluidic array systems. Anal. Chem. 2007, 79, 3504-3508. 